\section{Analyse, quantitative toxikologische}

C. Vidal ${ }^{1}$ und W.-R. Külpmann ${ }^{2}$

${ }^{1}$ Landeskriminalamt Niedersachsen, Dezernat 53 „Chemie“, Hannover, Deutschland

${ }^{2}$ Hannover, Deutschland

Englischer Begriff toxicological determination
Definition Quantitative Bestimmung der in einer Analysenprobe enthaltenen toxikologisch relevanten Substanzen meist mithilfe chemischer oder physikalisch-chemischer Verfahren.

Beschreibung. Die quantitative toxikologische $>$ Analyse erfasst bevorzugt die Konzentration von toxikologisch relevanten Substanzen im Blut bzw. Plasma. Sie erlaubt damit häufig einen Rückschluss auf die Bedeutung der Substanzen für den aktuellen Gesundheitszustand des Patienten. Die Bestimmungen erfordern sehr spezifische Analysenverfahren. 